\title{
Extracellular ATP is generated by ATP synthase complex in adipocyte lipid rafts
}

\author{
Bong-Woo Kim ${ }^{1}$, Hyo-Jung Choo ${ }^{1}$, \\ Joong-Won Lee ${ }^{1}$, Ji-Hyun $\mathrm{Kim}^{1}$ \\ and Young-Gyu Ko ${ }^{1,2}$ \\ ${ }^{1}$ Graduate School of Life Sciences and Biotechnology \\ Korea University, Seoul 136-701, Korea \\ ${ }^{2}$ Corresponding author: Tel, 82-2-3290-3453; \\ Fax, 82-2-927-9028; E-mail, ygko@korea.ac.kr \\ Accepted 18 October 2004
}

Abbreviations: Apo-Al, apolipoprotein Al; CCCP, carbonyl cyanide $m$-chlorophenylhydrazone; CTB, cholera toxin $B$; EMAP II, endothelial and monocyte-activating polypeptide II; PPAR $\gamma$, peroxisome proliferators-activated receptor $\gamma$; VDAC, voltage-dependent anion channels

\begin{abstract}
Mitochondrial biogenesis is known to accompany adipogenesis to complement ATP and acetyl-CoA required for lipogenesis. Here, we demonstrated that mitochondrial proteins such as ATP synthase $\alpha$ and $\beta$, and cytochrome $c$ were highly expressed during the 3T3-L1 differentiation into adipocytes. Fully-differentiated adipocytes showed a significant increase of mitochondria under electron microscopy. Analysis by immunofluorescence, cellular fractionation, and surface biotinylation demonstrated the elevated levels of ATP synthase complex found not only in the mitochondria but also on the cell surface (particularly lipid rafts) of adipocytes. High rate of ATP (more than $30 \mu \mathrm{M}$ ) synthesis from the added $A D P$ and $P_{i}$ in the adipocyte media suggests the involvement of the surface ATP synthase complex for the exracellular ATP synthesis. In addition, this ATP synthesis was significantly inhibited in the presence of oligomycin, an ATP synthase inhibitor, and carbonyl cyanide $m$-chlorophenylhydrazone (CCCP), an ATP synthase uncoupler. Decrease of extracellular ATP synthesis in acidic but not in basic media further indicates that the surface ATP synthase may also be regulated by proton gradient through the plasma membrane.
\end{abstract}

Keywords: adipocytes; ATP synthase complex; membrane microdomains; mitochondria

\section{Introduction}

An emergence of pluralistic function of a given protein is becoming a common finding. The multifunctionality of a protein can be achieved depending on its cellular localization, secretion, expression, oligomerization, ligand/substrate concentration, binding sites and complex formation (Jeffery, 1999). Many mitochondrial proteins are found in other unexpected cellular organelles. A recent proteomic analysis of integral plasma membrane protein shows many mitochondrial proteins such as ATP synthase, NADH dehydrogenase, and cytochrome $c$ oxidase, suggesting that ATP-generating mitochondrial proteins might have their novel functions in the plasma membrane (Zhao et al., 2004).

ATP synthase complex has been reported to have ability to recruit different ligands on the cell surface. ATP synthase complex associates with angiostatin, endothelial and monocyte-activating polypeptide II (EMAPII), and apolipoprotein $\mathrm{Al}$ (Apo-Al) that require cell surface receptors for their functions (Moser et al., 1999; Chang et al., 2001; Moser et al., 2001; Martinez et al., 2003). Since anti-ATP synthase $\alpha$ and $\beta$ antibodies abolish the function of angiostatin, EMAP II, and Apo-Al, the ATP synthase complex might be the receptors for these ligands. Alternatively, the surface ATP synthase complex might be involved in extracellular ATP generation because extracellular ATP generation is inhibited by treating endothelial cells with anti-ATP synthase $\alpha$ antibody or ATP synthase inhibitors (Moser et al., 1999; Arakaki et al., 2003).

Lipid rafts composed of cholesterol and sphingolipids concentrate various receptors and their downstream molecules to facilitate different cellular signal transductions on the plasma membrane (Anderson, 1998; Brown and London, 1998; Galbiati et al., 2001; Anderson and Jacobson, 2002; Capanni et al., 2003; $\mathrm{Ha}$ et al., 2003; Munro, 2003). The lipid rafts have been isolated from different mammalian cells using their distinct biochemical properties such as detergent insolubility and low density (Brown, 1992). Many lipid raft proteins have been identified by subproteomic analysis using liquid chromatography and tandem mass spectrometry as well as two-dimensional elec- 
trophoresis (Calvo et al., 2000; Bini et al., 2003; Foster et al., 2003; Li et al., 2003; Blonder et al., 2004; Sprenger et al., 2004). In addition to various receptors and signaling molecules, many mitochondrial proteins such as prohibitin, voltage-dependent anion channels (VDACs,) and ATP synthase complex have been annotated to the lipid rafts (Bini et al., 2003; Foster et al., 2003; Li et al., 2003; Sprenger et al., 2004). Since these proteins are repetitively found in the plasma membrane and lipid rafts that are highly purified, these proteins might not be contaminants from mitochondria.

Adipose tissue is not only an energy reservoir with lipid droplets composed of triacylglycerides and cholesterol but also an important endocrine organ to regulate insulin sensitivity, food intake, and wholebody energy metabolism by producing and releasing cytokines such as tumor necrosis factor (TNF), adipokine, resistin, and leptin (Evans et al., 2004). Since insulin stimulates lipid biosynthesis with acetyl-CoA that is synthesized in the mitochondria, lipogenesis is accompanied with mitochondrial biogenesis (WilsonFritch et al., 2003). Here, we showed that the expression of ATP synthase complex, and mitochondrial biogenesis were highly increased during adipogenesis. The increased ATP synthase complex was found in lipid rafts as well as in the mitochondria, suggesting that ATP synthase might have its specific function in the plasma membrane. Finally, the inhibition of high rate of extracellular ATP synthesis in the adipocytes by oligomycin, an ATP synthase inhibitor, and CCCP, an ATP synthase uncoupler suggest that the surface ATP synthase might be involved in extracellular ATP generation in adipocytes.

\section{Materials and Methods}

\section{Antibodies}

Anti-ATP synthase $\alpha$ and $\beta$ antibodies, and rhodamine conjugated-cholera toxin $B$ (CTB) were obtained from molecular probes. Anti-insulin receptor $\beta$, caveolin-1, and flotillin-1 antibodies were from BD Biosciences. Anti-cytochrome $\mathrm{c}$, and PPAR $\gamma$ antibodies were purchased from Santa Cruz.

\section{Adipocyte differentiation}

3T3-L1 fibroblasts were purchased from ATCC, and grown in DMEM supplemented with $1 \%$ penicillin/ streptomycin (Jeil Biosciences Inc.), and 10\% calf serum (Jeil Biosciences Inc.) in $5 \% \mathrm{CO}_{2}$ incubator at $37^{\circ} \mathrm{C}$. Three-day-postconfluent 3T3-L1 fibroblasts were differentiated into adipocytes by incubating with DMEM supplemented with the same antibiotics, 10\% FBS, $0.25 \mathrm{mM}$ 3-isobutyl-1-methylxanthine (Sigma),
$0.25 \mu \mathrm{M}$ dexamethasone (Sigma), and $200 \mathrm{nM}$ insulin (Sigma). After $48 \mathrm{~h}$, the adipocytes were continuously incubated with fresh DMEM containing $10 \%$ fetal bovine serum (FBS) and $200 \mathrm{nM}$ insulin. The adipocytes were re-fed with DMEM containing $10 \%$ FBS every $48 \mathrm{~h}$.

\section{Preparation of human adipocyte}

Dissected fat pads from human were immersed in Krebs Ringer Hepes (KRH) buffer containing $120 \mathrm{mM}$ $\mathrm{NaCl}, 4 \mathrm{mM} \mathrm{KH} \mathrm{PO}_{4}, 17 \mathrm{mM} \mathrm{MgSO}_{4}, 1 \mathrm{mM} \mathrm{CaCl}_{2}$, $10 \mathrm{mM} \mathrm{NaHCO} 3,30 \mathrm{mM}$ Hepes, $2.5 \mathrm{mM}$ D-glucose, $200 \mathrm{nM}$ adenosine, and 2\% albumin. Adipose tissues were minced and digested with $2.5 \mathrm{mg} / \mathrm{ml}$ collagenase (Sigma) for $1 \mathrm{~h}$ at $37^{\circ} \mathrm{C}$ in shaking water bath. The suspension was centrifuged and the floating adipocytes were collected and filtered through 400-um nylon mesh to remove tissue pieces. The cell suspension was then centrifuged at $400 \mathrm{~g}$ for $1 \mathrm{~min}$. Adipocytes were collected from the supernatant of the suspension and resuspended in cold PBS. The centrifugation was repeated 3 times.

\section{Isolation of mitochondria and plasma membrane}

Plasma membrane was isolated according to the method of Hubbard et al. (1983) with small modifications. Differentiated 3T3-L1 adipocytes were washed twice with TES buffer $(20 \mathrm{mM}$ Tris $\mathrm{HCl}, 1 \mathrm{mM}$ EDTA, $8.7 \%$ sucrose; $\mathrm{pH} 7.4$ ) and scraped. Cells were homogenized in TES buffer in a tight Dounce homogenizer (Kontes). The homogenized cells were centrifuged at $12,000 \mathrm{rpm}$ in a SW55Ti rotor (Beckman) for $30 \mathrm{~min}$. The pellet was resuspended in TES buffer, loaded on $50 \%$ sucrose and $38.5 \%$ sucrose cushion, and centrifuged at $100,000 \mathrm{~g}$ in a SW55Ti rotor for $60 \mathrm{~min}, 4^{\circ} \mathrm{C}$. Plasma membrane was collected from the top of the sucrose cushion, resuspended in TES buffer, and repelleted by centrifugation at $31,000 \mathrm{~g}$ for $60 \mathrm{~min}, 4^{\circ} \mathrm{C}$. Mitochondria were collected from the bottom of the sucrose cushion, resuspended in TES buffer, and repelleted by centrifugation at $5,000 \mathrm{~g}$ for $60 \mathrm{~min}, 4^{\circ} \mathrm{C}$.

\section{Immunoblotting and immunofluorescence}

Preadipocytes and adipocytes were lysed with SDS lysis buffer $(25 \mathrm{mM}$ Tris- $\mathrm{HCl}, \mathrm{pH} 7.4,150 \mathrm{mM} \mathrm{NaCl}$, $1 \%$ Triton $\mathrm{X}-100,1 \%$ sodim deoxy cholate, $0.1 \%$ SDS, $1 \mathrm{mM}$ EDTA, $1 \mathrm{mM}$ PMSF, and protease cocktail (Roche)) for $20 \mathrm{~min}$ at $4^{\circ} \mathrm{C}$. After microcentrifugation at $14,000 \mathrm{rpm}, 10 \mathrm{~min}, 4^{\circ} \mathrm{C}$, the whole cell lysates (supernatant) were separated by SDS-polyacrylamide gels and transferred to a nitrocellulose membrane. Antigens were visualized by sequential treatment with specific antibodies, HRP-conjugated secon- 
dary antibodies, and an enhanced chemiluminescence substrate kit.

For immunofluorescence, cells were fixed with $10 \%$ formaldehyde in PBS for $20 \mathrm{~min}$. The cells were incubated with blocking buffer (5\% BSA in PBS), and primary antibodies. The primary antibodies were detected with fluorescein-conjugated secondary antibodies. Also cells were incubated with rhodamine-conjugated CTB. Cells were observed with a Bio-Rad confocal microscope (MRC 1024).

\section{Electron microscopy}

3T3-L1 preadipocytes and adipocytes were fixed for $1 \mathrm{~h}$ at $4^{\circ} \mathrm{C}$ in $0.1 \mathrm{M}$ sodium phosphate buffer $(\mathrm{pH}$ 7.4) containing $2 \%$ paraformaldehyde- $2.5 \%$ glutaraldehyde mixture. Cells were harvested with cell lifter and washed with same buffer, centrifuged at $3,000 \mathrm{rpm}$ for 5 mins. The pellets were post-fixed for $1 \mathrm{~h}$ in the same buffer containing $1 \% \mathrm{OsO}_{4}$, subsequently dehydrated using graded acetone, and embedded in Epon-Araldite. Thick and thin sections were prepared on RMC MT-X ultramicrotome. The sectioned thick tissues were stained with $1 \%$ toluidin blue-borax solution, mounted on copper grids, and double-stained with uranyl acetate. The grids were examined in JEM 100 CX-II electron microscope.

\section{Surface biotinylation}

Cell monolayers were biotinylated with $0.2 \mathrm{mg} / \mathrm{ml}$ EZ-link-sulfo-NHS-LC-biotin (Pierce) prepared in 10 $\mathrm{mM}$ Hepes, $\mathrm{pH} 8.0,150 \mathrm{mM} \mathrm{NaCl}, 0.2 \mathrm{mM} \mathrm{MgCl}_{2}$, and $0.2 \mathrm{mM} \mathrm{CaCl}_{2}$ at $4{ }^{\circ} \mathrm{C}$ for $30 \mathrm{~min}$. The reaction was terminated by washes with $10 \mathrm{mM}$ Tris $\cdot \mathrm{HCl}(\mathrm{pH}$ 7.5). The biotinylated cells were lysed with octylglucoside lysis buffer containing $1 \%$ Triton X-100, 60 mM $n$-octyl $\beta$-D-glucopyranoside, $150 \mathrm{mM} \mathrm{NaCl}, 25$ $\mathrm{mM}$ Tris $(\mathrm{pH} 7.4), 1 \mathrm{mM}$ EDTA, and protease cocktail at $4^{\circ} \mathrm{C}$ for $30 \mathrm{~min}$. After microcentrifugation at $4^{\circ} \mathrm{C}$ for $20 \mathrm{~min}$, the supernatant with $700 \mu \mathrm{g}$ of protein was reacted with $50 \mathrm{ml}$ of avidin-conjugated agarose beads at $4^{\circ} \mathrm{C}$ for $12 \mathrm{~h}$. The beads were washed four times with octylglucoside lysis buffer. The precipitated proteins were resolved on 10\% SDS-PAGE, transferred to nitrocellulose membranes, and analyzed by immunoblotting with ATP synthase $\alpha$ and $\beta$ antibodies.

\section{Lipid raft isolation}

Four 100-mm dishes of differentiated 3T3-L1 adipocytes, or $500 \mu$ cell volume of human adipocytes were mixed with in $1 \mathrm{ml}$ of lysis buffer (1\% Brij 35 , $25 \mathrm{mM}$ Hepes, pH 6.5, $150 \mathrm{mM} \mathrm{NaCl}, 1 \mathrm{mM}$ EDTA, $1 \mathrm{mM}$ PMSF, and protease cocktail), homogenized 20 times with a tight Dounce homogenizer (Kontes), and incubated at $4^{\circ} \mathrm{C}$ for $30 \mathrm{~min}$. The extract was mixed with $1 \mathrm{ml}$ of $2.5 \mathrm{M}$ sucrose, transferred to an SW41Ti centrifuge tube, and overlaid with $6 \mathrm{ml}$ of $30 \%$ sucrose solution and $4 \mathrm{ml}$ of $5 \%$ sucrose solution containing $25 \mathrm{mM}$ Hepes, $\mathrm{pH} 6.5$, and $150 \mathrm{mM} \mathrm{NaCl}$. The discontinuous sucrose gradients were centrifuged for $18 \mathrm{~h}$ at $4^{\circ} \mathrm{C}$ in an SW41Ti rotor at $39,000 \mathrm{rpm}$. The gradient was fractionated into 12 fractions from the bottom to the top.

\section{Quantification of ATP by bioluminescent luciferase assay}

Extracellular ATP was measured following the protocol described previously (Arakaki et al., 2003). 3T3-L1 preadipocytes were seeded and differentiated in 24-well plates and confluent 3T3-L1 adipocytes were used for cell surface ATP synthesis. Cells were washed with Hepes buffer (10 mM Hepes, $\mathrm{pH} 7.4$, $150 \mathrm{mM} \mathrm{NaCl}$ ) three times and were incubated with buffer only or $0.3 \mathrm{ml}$ of Hepes buffer containing 200 $\mu \mathrm{M}$ ADP, $20 \mathrm{mM}$ potassium phosphate $\left(P_{\mathrm{i}}\right), 2 \mathrm{mM}$ $\mathrm{MgCl}_{2}$ at room temperature. After $10-480 \mathrm{~s}$, the extracellular media were removed and supernatants $(20 \mu l)$ were used for the determination of extracellular ATP content. ATP levels were measured by the bioluminescence assay according to the protocol provided with an ATP determination kit (Molecular probes).

\section{Results}

\section{ATP synthase complex is highly expressed during adipogenesis}

Recent proteomic analysis reveals that adipogenesis is accompanied with mitochondrial biogenesis as evidenced by significant increase of mitochondrial proteins in the differentiated 3T3-L1 cells. After postadipocyte differentiation, there is a significant increase of known mitochondrial proteins involved in the lipid biosynthesis: carnitine aceyltransferase, succinyl CoA ligase, pyruvate decarboxylase, and malate dehydrogenase (Wilson-Fritch et al., 2003). To better understand an effect on the ATP synthase complex, an essential mitochondrial machinery for ATP synthesis during adipogenesis, 3T3-L1 preadipocytes were differentiated into adipocytes for $0,2,4,6$, and 8 days, and the cell lysates were analyzed by immunoblotting with anti-ATP synthase $\alpha$ and $\beta$, cytochrome $c$, peroxisome proliferators-activated receptor $\gamma$ (PPAR $\gamma)$, and $\beta$-actin antibodies. As shown in Figure 1 , mitochondrial proteins such as ATP synthase $\alpha$ and $\beta$ and cytochrome $c$ were highly expressed in fullydifferentiated adipocytes with high level of PPAR $\gamma$, an adipocyte differentiation marker, suggesting that $\mathrm{mi}$ - 
tochondrial proteins are highly expressed during adipocyte differentiation.

For additional evidence of the increased mitochondrial biogenesis during adipogenesis, the morphological features of preadipocytes and adipocytes

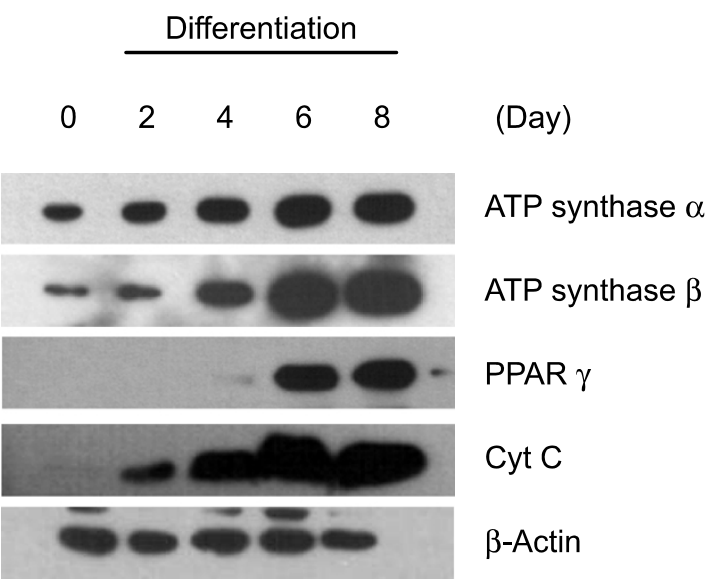

Figure 1. The expression of ATP synthase complex is highly induced during 3T3-L1 adipogenesis. 3T3-L1 preadipocytes were differentiated to adipocytes for $0,2,4,6$, and 8 days. The differentiated adipocytes were lysed with SDS lysis buffer, and the lysates were analyzed by immunoblotting with anti-ATP synthase $\alpha$ and $\beta$, cytochrome $c$, PPAR $\gamma$, and insulin receptor antibodies. were examined under electron microscopy. Mitochondria in preadipocyte were rare, irregular, and smallsized (Figure 2). On the contrary, adipocyte mitochondria were densely packed, elongated, and largesized. In addition, abundant lamellar cristae, which were rare in preadipocytes, were observed in fullydifferentiated adipocytes, suggesting that mitochodria in adipocytes appear to be functional. The number of mitochondria was estimated by counting in seven images containing whole-cell profiles sectioned through the middle of the nucleus. A preadipocyte had 7 mitochondria while an adipocyte 132 in average (data not shown).

\section{Surface expression of ATP synthase complex in adipocytes}

In previous reports, ATP synthase complex has been found in the surface of endothelial cells and hepatocyte and associated with different ligands such as angiostatin, EMAPII, and Apo-AI. Such findings of ATP synthase complex in both the plasma membrane and in mitochondria presented yet undefined interrelationship of two ATP synthase complexes. In order to address this issue, preadipocyte and adipocyte were immunostained with anti-ATP synthase $\alpha$ and $\beta$ antibodies. It should be noted that permeabilization step was omitted to see only immunofluorescent
Preadipocyte

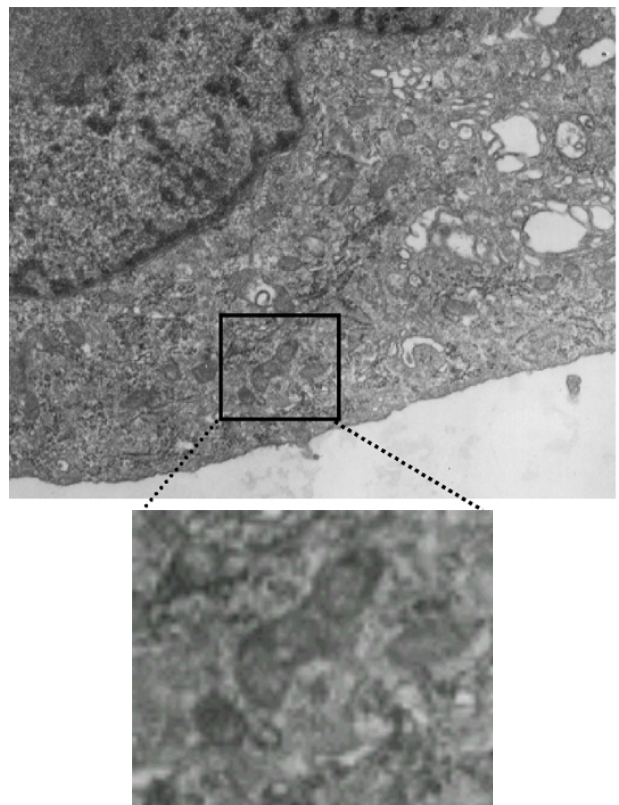

Adipocyte

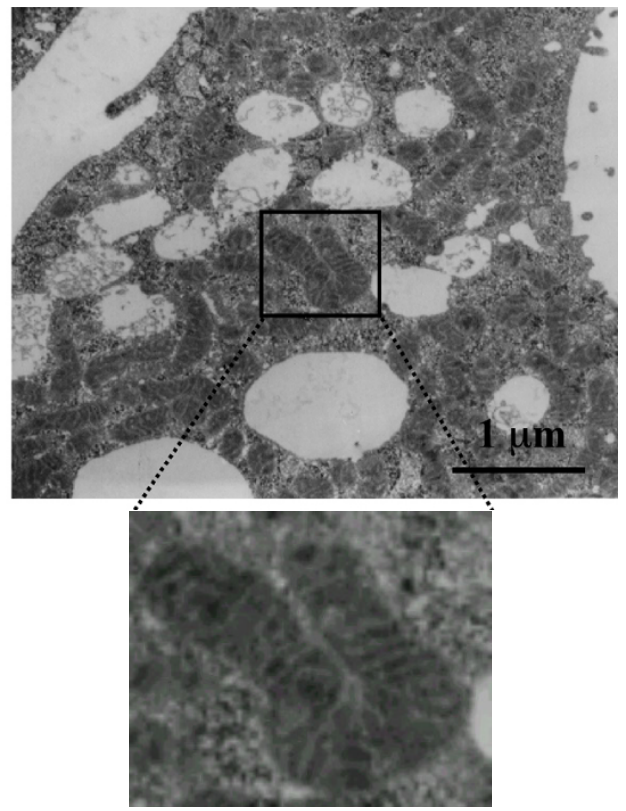

Figure 2. Mitochondria are packed full in fully-differentiated adipocytes. On day 8 of differentiation, 3T3-L1 cells were used as fully-differentiated adipocytes. 3T3-L1 preadipocytes and adipocytes were prepared for electron microscopy (magnification, $\times 25,000$ ). Insets in upper panels were enlarged in bottom panels to see mitochondrial morphology in detail. 
signal from the cell surface. Figure $3 A$ showed that ATP synthase $\alpha$ and $\beta$ were expressed in the cell surface of preadipocytes and adipocytes. However, the immunostaining signal was stronger in adipocytes than in preadipocytes, suggesting that more ATP synthase complex is expressed in the cell surface of adipocytes than that of preadipocytes.

In order to confirm the surface expression of ATP synthase complex in preadipocytes and adipocytes, both cells were labeled with membrane impermeable biotin. The biotin-labeled surface proteins were precipitated with avidin-conjugated agarose beads. The precipitates were immunoblotted with anti-ATP synthase $\alpha$ and $\beta$ antibodies. Both ATP synthase $\alpha$ and $\beta$ were biotinylated (Figure $3 \mathrm{~B}$ ), demonstrating that they are indeed expressed on the cellular surface. However, since more ATP synthase $\alpha$ and $\beta$ were appeared to be biotinylated in adipocytes than in preadipocytes, additional experiments were carried out to clarify that ATP synthase complex is highly expressed on the cell surface during adipogenesis. Plasma membrane and mitochondria proteins in preadipocytes and adipocytes were isolated and analyzed by immunoblotting with anti-ATP synthase $\alpha$ and $\beta$, cytochrome $c$, and insulin receptor antibodies. Cytochrome $c$ was mainly found in mitochondria fraction whereas insulin receptor only in the plasma membrane, assuring that there was no cross-contamination between plasma membrane and mitochondria proteins during their preparation. As shown in Figure
A

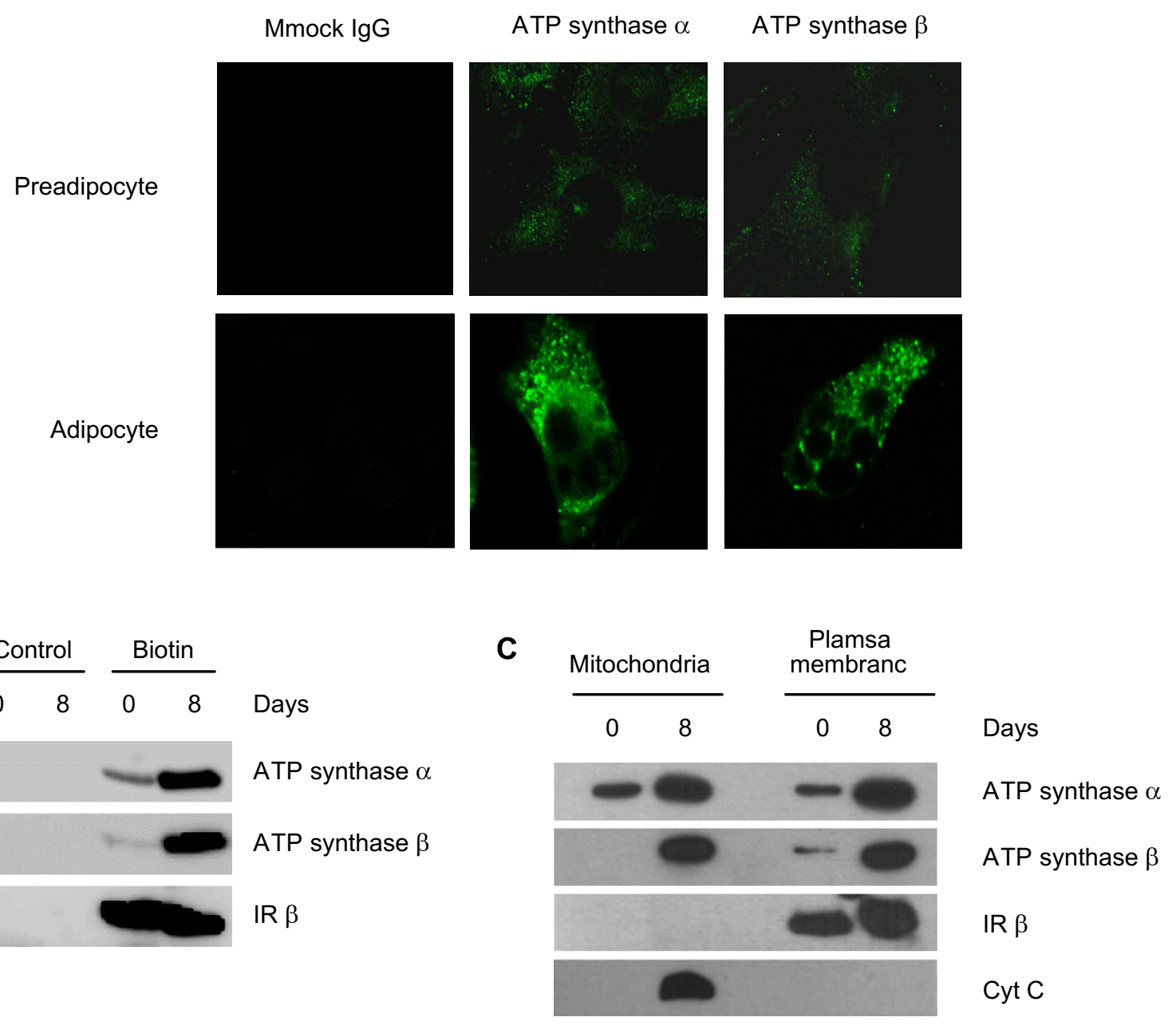

Figure 3. ATP synthase complex is expressed on the outer leaflet of the plasma membrane. On day 8 of differentiation, 3T3-L1 cells were used as fully-diffentiated adipocytes. (A) 3T3-L1 preadipocytes and adipocytes were immunostained with anti-ATP synthase $\alpha$ and $\beta$ antibodies, and mock lgG. It should be noted that permeabilization step was skipped out for visualizing the surface expression of ATP synthase complex. Scale bar $=10 \mu \mathrm{m}$. (B) 3T3-L1 preadipocytes and adipocytes were labeled with membrane-impermeable biotin and lysed with lysis buffer. The lysates were precipitated with avidin-conjugated agarose beads and the precipitates were analyzed with anti-ATP synthase $\alpha$ and $\beta$ antibodies. (C) Plasma membrane, and mitochondria were prepared from 3T3-L1 preadipocytes and adipocytes. Each protein $(5 \mu \mathrm{g})$ from plasma membrane, and mitochondria were analyzed by immunoblotting with anti-ATP synthase $\alpha$ and $\beta$ antibodies. IR, insulin receptor. 
3C, more ATP synthase $\alpha$ and $\beta$ were found in the plasma membrane and mitochondria in adipocytes than preadipocytes, supporting that adipogenesis is accompanied with high surface expression of ATP synthase complex.

\section{ATP synthase complex is found in lipid rafts}

ATP synthase $\alpha$ and $\beta$ might be in the plasma membrane rafts because of its patchy and punctate staining pattern that is typically shown in raft proteins. We observed the co-localization of ATP synthase $\alpha$ or $\beta$ with cholera toxin $B$ (CTB) that is a gangliosidebinding protein used as lipid raft landmarker. Figure 4A showed that ATP synthase $\alpha$ and $\beta$ were indeed co-localized with CTB, indicating that ATP synthase complex is localized in lipid rafts. It should be noted that the cells were not permeabilized for immunofluorescence.

In order to make certain that ATP complex is a lipid raft protein, we isolated detergent-resistant lipid rafts from the differentiated 3T3-L1, and human adipocytes, and analyzed them by immunoblotting with antiATP synthase $\alpha$ and $\beta$, cytochrome $c$, insulin receptor, caveolin-1, and flotillin-1 antibodies. Since cytochrome $c$ was found only in the bottom fractions whereas insulin receptor, caveolin-1, and flotillin-1 were present in raft fractions, there was little mitochondrial contamination in lipid raft fractions (Figure 4B). As shown in Figure 4B, lipid raft fractions
A
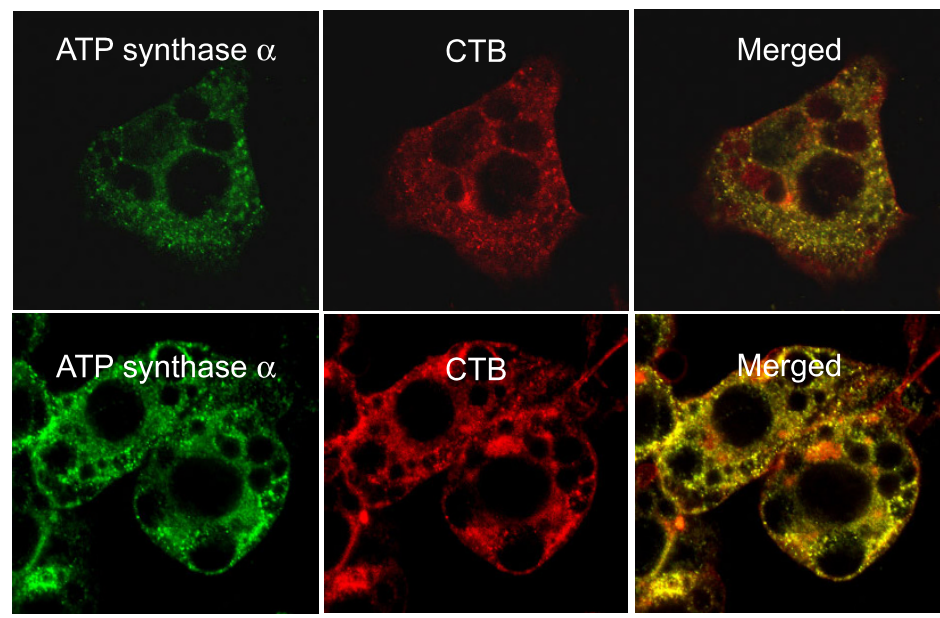

B

Lipid Rafts

$\mathrm{P} 12234566 \overline{789} 101112$

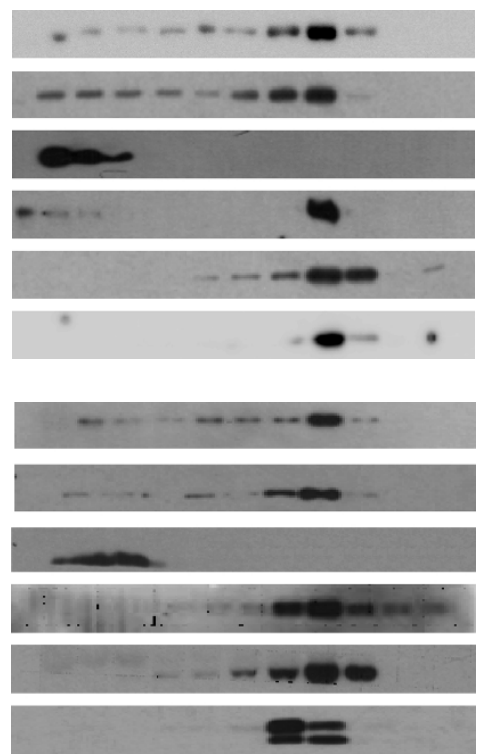

ATP synthase $\alpha$

ATP synthase $\beta$

Cytochrome C

IR $\beta$

Flotillin-1

Caveolin-1

ATP synthase $\alpha$

ATP synthase $\beta$

Cytochrome C

IR $\beta$

Flotillin-1

Caveolin-1

3T3 L1 adipocyte

Figure 4. ATP synthase $\alpha$ and $\beta$ are enriched in lipid rafts of adipocytes. (A) ATP synthase $\alpha$ and $\beta$ are co-localized with CTB in fully-differentiated adipocytes, indicating that the complex is present in the plasma membrane rafts. 3T3-L1 adipocytes were immunostained with anti-ATP synthase $\alpha$ or $\beta$ antibody and rhodamine-conjugated CTB. It should be noted that permeabilization step was skipped out for immunofluorescence. Scale bar = 10 um. (B) Detergent-resistant lipid rafts were isolated from 3T3-L1 adipocytes and human adipocytes as outlined in Materials and Methods. Each fraction from the sucrose gradient was blotted with anti-ATP synthase $\alpha$, and $\beta$, cytochrome c, insulin receptor (IR), flotillin-1, and caveolin-1 antibodies. 
contained large portions of ATP synthase $\alpha$ and $\beta$, indicating that ATP synthase complex is present in the detergent-resistant lipid rafts of mouse and human adipocytes.

\section{ATP synthase is required for the generation of extracellular ATP}

In order to explore the function of surface ATP synthase, ATP content was determined from adipocyte media after treating the cells with ADP and inorganic phosphate $\left(\mathrm{P}_{\mathrm{i}}\right)$.

Extracellular ATP content had greatly increased with a biphasic velocity with an inflection point at 30 seconds after $A D P$ and $P_{i}$ addition to adipocyte media and reached a plateau level $(30 \mu \mathrm{M})$ at $8 \mathrm{~min}$ that was continuously maintained for longer time (Figure $5 A)$. The result of the rapid generation of the extracellular ATP upon addition of substrates, ADP and $P_{i}$ and no lag period strongly suggest a direct catalysis by the exposed surface ATP synthase. The extracellular ATP level was extremely low (less than 10 $n M)$ in the absence of substrate ADP and $P_{i}$.

The efficiency of ATP synthase catalysis was further examined by rapid response of ATP synthase inhibition. Adipocytes were pretreated with oligomycin
$(10 \mu \mathrm{g} / \mathrm{ml})$ or CCCP $(0.4 \mu \mathrm{g} / \mathrm{ml})$ for $5 \mathrm{~min}$. Extracellular ATP content from media was measured 1 minute after adding $A D P$ and $P_{i}$. As shown in Figure $5 \mathrm{~B}$, extracellular ATP synthesis was inhibited by about $53 \%$ in the presence of oligomycin, presumably supporting that catalysis by the exposed cellular ATP synthase is affected. Since ATP synthase complex uses proton gradient to generate ATP in mitochondria, it is tempting to speculate that surface ATP synthase also requires proton gradient for synthesizing extracellular ATP. Thus, the content of extracellular ATP was measured in the presence of carbonyl cyanide m-chlorophenylhydrazone (CCCP), a mitochondrial uncoupler or proton gradient destroyer. CCCP decreased extracellular ATP content by about $46 \%$, suggesting that proton gradient is required for extracellular ATP generation. Figure 5B showed that extracellular ATP generation was not affected by DMSO that is a carrier solution solubilizing oligomycin and CCCP.

Since ATP synthase complex was exposed to extracellular space (Figure 3), there could be a proton gradient from cytoplasm to extracellular space for generating extracellular ATP. Cell media should be more basic than cytosol to form a proton gradient through the plasma membrane. In order to address

\section{A}

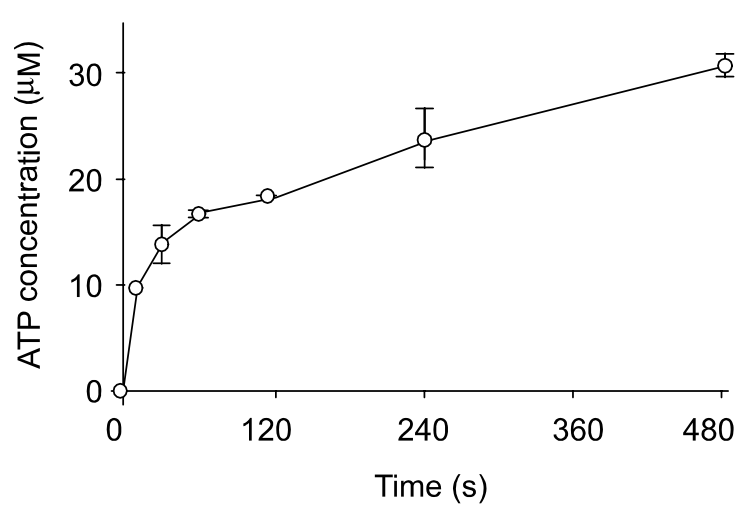

B

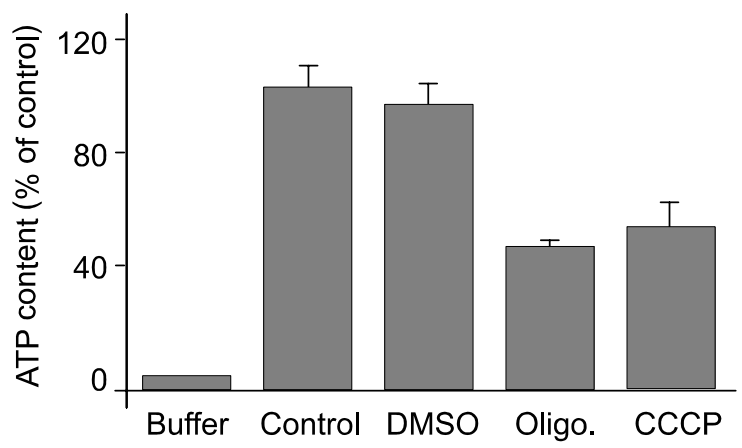

C

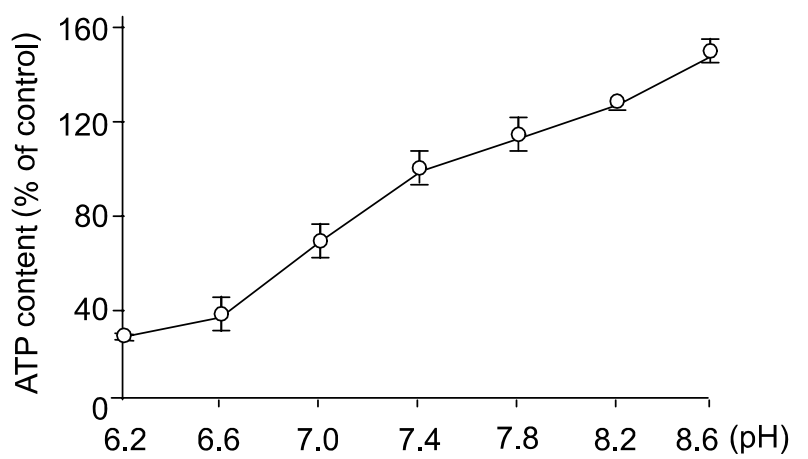

Figure 5. Extracellular ATP synthesis by surface ATP synthase complex. On day 8 of differentiation, 3T3-L1 cells were used as fullydiffentiated adipocytes. (A) Extracellular ATP synthesis was initiated by the addition of ADP and $P_{i}$ to a culture of adipocytes. At the indicated time, the extracellular media were collected, and the ATP content in those pools was determined. (B) The effects of oligomycin and CCCP in extracellular ATP synthesis. Adipocytes were pretreated with $0.5 \%$ DMSO, oligomycin $10 \mu \mathrm{g} / \mathrm{ml})$, or CCCP $(0.4 \mu \mathrm{g} / \mathrm{ml})$ for 5 min. Extracellular ATP content from media was measured 1 min after adding ADP and Pi. Control indicates the extracellular ATP content generated in the absence of any drug. DMSO, dimethyl sulfphoxide; oligo., oligomycin; CCCP, carbonyl cyanide m-chlorophenylhydrazone. (C) The pH effect of cell media in extracellular ATP synthesis. Adipocytes were washed twice, and then extracellular ATP content was measured 1 min after adding ADP and $\mathrm{P}_{\mathrm{i}}$ into media of different $\mathrm{pH}$. Control indicates the extracellular ATP content generated in $\mathrm{pH} 7.4$. 
the issue, we incubated adipocytes in acidic, neutral, or basic condition and then measured extracellular ATP. As shown in Figure 5C, less extracellular ATP was generated in less basic condition. Meanwhile, more extracellular ATP was synthesized in more basic condition. Taken together all these data in Figure 5, we can conclude that surface ATP synthase is required for extracellular ATP synthesis.

\section{Discussion}

After cellular exposure to insulin, lipogenesis is activated in adipocytes. Thus, many enzymes for lipid biosynthesis are highly up-regulated and activated in adipocytes. The lipid biosynthesis requires mitochondrial and cytoplasmic enzymes. Mitochondria are necessary for the formation of acetyl-CoA and NADH that are essential components for lipid biosynthesis. Thus, adipogenesis should be accompanied with mitochondrial biogenesis. Here, we demonstrate that mitochondria are extravagantly generated during adipogenesis by immunoblotting and electron microscopy. Compared to preadipocyte mitochondria, adipocyte mitochondria have more elongated and electrondense morphology (Figure 2). In addition, there is a tremendous increase of mitochondria number during adipogenesis; adipocyte has 19 times more mitochondria than preadipocyte. With their large number and elongated morphology, adipocyte mitochondria could be active for synthesizing ATP and NADH, and for producing and transferring more acetyl-CoA to cytosol to synthesize lipid. Thus, many mitochondrial proteins are highly up-regulated during adipogenesis. We also demonstrated that cytochrome $c$ and ATP synthase complex are highly expressed in adipocytes (Figure 1), suggesting that the adipocytes are active for oxidative respiration to generate NADH and ATP.

Some of mitochondrial proteins such as prohibitin, VDACs, ATP/ADP exchanger, and ATP synthase complex have been described in association with the plasma membrane or lipid rafts. Among these proteins, ATP synthase complex has been proven to be localized in the plasma membrane of human umbilical vein endothelial cells (HUVECs), and hepatocytic HepG2 cells (Moser et al., 1999; Moser et al., 2001; Martinez et al., 2003). We also found that the ATP synthase complex was highly expressed on the cell surface of $\mathrm{C} 2 \mathrm{C} 12$ myocytes (data not shown) as well as 3T3-L1 adipocytes (Figure 3). Interestingly, endothelial cells, hepatocytes, and myocytes, which express ATP synthase complex on their cell surface, respond with angiostatin to inhibit cellular proliferation (Moser et al., 2001; Wajih et al., 2003). Thus, angiostatin might bind to the cell surface through ATP synthase complex because its binding to the cell surface was abolished by competing with anti-ATP synthase antibody.

Martinez et al. advocated that Apo-Al binds to surface ATP synthase complex in hepatocytes to induce the uptake of high density lipoprotein (HDL) (Martinez et al., 2003). Since the uptake of Apo-Alcontaining HDL is active in adipocytes (Dagher et al., 2003), and ATP synthase complex is expressed on the surface of adipocytes (Figure 3 ), we tested the HDL uptake after neutralizing ATP synthase complex with anti-ATP synthase $\beta$ antibody that previously used for preventing surface binding of Apo-Al (Martinez et al., 2003). From the experiments, HDL uptake was significantly inhibited by anti-scavenger receptor I (SR-BI) antibody but not by anti-ATP synthase $\beta$ antibody (data not shown), suggesting that the surface ATP synthase of adipocytes is not involved in HDL uptake.

The major function of surface ATP synthase might be to synthesize extracellular ATP. Indeed, extracellular ATP generation is abolished in human endothelial cells when neutralized with its antibody (Moser et al., 2001). Since the extracellular ATP generation is dramatically decreased by treating human endothelial cells with ATP synthase inhibitors such as efrapeptins, piceatannol, and resveratrol (Arakaki et al., 2003), surface ATP synthase is necessary for ATP synthesis on the cell surface. We also measured ATP content in culture media of adipocytes after adding ADP plus $\mathrm{P}_{\mathrm{i}}$. Eight minutes after treating adipocytes with ADP plus $P_{i}$, more than $30 \mu \mathrm{M}$ extracellular ATP was found. Since the extracellular ATP synthesis was prevented by oligomycin, the extracellular ATP generation might require surface ATP synthase complex. Interestingly, the extracellular ATP generation was abolished when proton gradient was disrupted by treating adipocytes with CCCP or in acidic condition, suggesting that proton gradient through the plasma membrane is necessary for activating surface ATP synthase complex.

The extracellular ATP and nucleotides recognize and activate their receptors called $\mathrm{P} 2$ receptors (Schwiebert and Zsembery, 2003). P2 receptors are divided into two classes, ionotropic $P 2 X\left(P 2 X_{1-7}\right)$, and $G$ protein-coupled $P 2 Y\left(P 2 Y_{1-11}\right)$ receptors. ATP and other nucleotides modulate $\mathrm{Ca}^{2+}$ influx through $\mathrm{P} 2 \mathrm{X}$ receptors. Unlike $\mathrm{P} 2 \mathrm{X}$ receptors, $\mathrm{P} 2 \mathrm{Y}$ receptors activates $G_{q}$, phospholipase $C$, the generation of diacylglycerol and inositol triphosphate, increase of intracellular $\mathrm{Ca}^{2+}$, and protein kinase $\mathrm{C}$ or modulates $\mathrm{G}_{\mathrm{s}}$ or $\mathrm{G}_{\mathrm{i}}$, adenylyl cyclase, and resultant cAMP formation. Depending on the cell types, extracellular ATP ignites cellular responses such as cellular proliferation, muscle contraction and differentiation, inflammation, and neuronal communication (Bae and Ryu, 2001; Schand Zsembery, 2003). 
The extracellular ATP is rapidly dephosphorylated into ADP, AMP, and adenosine at the cell surface (Schwiebert and Zsembery, 2003). For example, ectoapyrases, ecto-ATPases, ecto-ADPases, b-5'-nucleotidase, and alkaline phosphatase are released from cells or bind to the outer leaflet of plasma membrane with glycosylphosphatidylinositol (GPI) anchor to hydrolyze the extracellular ATP. Interestingly, many ATP-hydrolyzing enzymes are found in lipid rafts (Strohmeier et al., 1997; Kittel et al., 1999; Ko et al., 1999). In addition, we demonstrate that lipid rafts concentrate ATP synthase complex, suggesting that lipid rafts are major plasma membrane compartments regulating ATP synthesis and hydrolysis.

\section{Acknowledgement}

This work was supported by a grant from the $21 \mathrm{C}$ frontier for the functional proteomics (FPG-02-A-5).

\section{References}

Anderson RG. The caveolae membrane system. Annu Rev Biochem 1998;67:199-225

Anderson RG, Jacobson K. A role for lipid shells in targeting proteins to caveolae, rafts, and other lipid domains. Science 2002;296:1821-5

Arakaki N, Nagao T, Niki R, Toyofuku A, Tanaka $H$, Kuramoto $Y$, Emoto $Y$, Shibata $H$, Magota $K$, Higuti $T$. Possible role of cell surface $H+-A T P$ synthase in the extracellular ATP synthesis and proliferation of human umbilical vein endothelial cells. Mol Cancer Res 2003;1:931-9

Bae YS, Ryu SH. ATP-induced focal adhesion kinase activity is negatively modulated by phospholipase D2 in PC12 cells. Exp Mol Med 2001;33:150-5

Bini L, Pacini S, Liberatori S, Valensin S, Pellegrini $M$, Raggiaschi R, Pallini V, Baldari CT. Extensive temporally regulated reorganization of the lipid raft proteome following T-cell antigen receptor triggering. Biochem J 2003;369:301-9

Blonder J, Hale ML, Lucas DA, Schaefer CF, Yu LR, Conrads TP, Issaq HJ, Stiles BG, Veenstra TD. Proteomic analysis of detergent-resistant membrane rafts. Proteomics 2004;25:1307-18

Brown DA, London E. Functions of lipid rafts in biological membranes. Annu Rev Cell Dev Biol 1998;14:111-36

Brown DA, Rose JK. Sorting of GPI-anchored proteins to glycolipid-enriched membrane subdomains during transport to the apical cell surface. Cell 1992;68:533-44

Calvo M, Enrich C. Biochemical analysis of a caveolaeenriched plasma membrane fraction from rat liver. Electrophoresis 2000;21:3386-95

Capanni C, Sabatelli P, Mattioli E, Ognibene A, Columbaro M, Lattanzi G, Merlini L, Minetti C, Maraldi NM, Squarzoni $S$. Dysferlin in a hyperCKaemic patient with caveolin 3 mutation and in $\mathrm{C} 2 \mathrm{C} 12$ cells after p38 MAP kinase inhibition.
Exp Mol Med 2003;35:538-44

Chang SY, Park SG, Kim S, Kang CY. Interaction of the C-terminal domain of $\mathrm{p} 43$ and the alpha subunit of ATP synthase. Its functional implication in endothelial cell proliferation. J Biol Chem 2001;277:8388-94

Dagher G, Donne N, Klein C, Ferre P, Dugail I. HDLmediated cholesterol uptake and targeting to lipid droplets in adipocytes. J Lipid Res 2003;44:1811-20

Evans RM, Barish GD, Wang YX. PPARs and the complex journey to obesity. Nat Med 2004;10:355-61

Foster LJ, de Hoog CL, Mann M. Unbiased quantitative proteomics of lipid rafts reveals high specificity for signaling factors. Proc Nat'l Acad Sci USA 2003;100:5813-8

Galbiati F, Razani B, Lisanti MP. Emerging themes in lipid rafts and caveolae. Cell 2001;106:403-11

Ha H, Kwak HB, Lee SW, Kim HH, Lee ZH. Lipid rafts are important for the association of RANK and TRAF6. Exp Mol Med 2003;35:279-84

Hubbard AL, Wall DA, Ma A. Isolation of rat hepatocyte plasma membranes. I. Presence of the three major domains. J Cell Biol 1983;96:217-9

Jeffery CJ. Moonlighting proteins. Trends Biochem Sci $1999 ; 24: 8-11$

Kittel A, Kaczmarek E, Sevigny J, Lengyel K, Csizmadia E, Robson SC. CD39 as a caveolar-associated ectonucleotidase. Biochem Biophys Res Commun 1999;262:596-9

Ko YG, Lee JS, Kang YS, Ahn JH, Seo JS. TNFa-mediated apoptosis is initiated in caveolae-like domains. J Immunol 1999:162:7217-23

Li N, Mak A, Richards DP, Naber C, Keller BO, Li L, Shaw AR. Monocyte lipid rafts contain proteins implicated in vesicular trafficking and phagosome formation. Proteomics 2003;3:536-48

Martinez LO, Jacquet S, Esteve JP, Rolland C, Cabezon E, Champagne E, Pineau T, Georgeaud V, Walker JE, Terce F, Collet X, Perret B, Barbaras R. Ectopic beta-chain of ATP synthase is an apolipoprotein A-I receptor in hepatic HDL endocytosis. Nature 2003;421:75-9

Moser TL, Stack MS, Asplin I, Enghild JJ, Hojrup P, Everitt L, Hubchak S, Schnaper HW, Pizzo SV. Angiostatin binds ATP synthase on the surface of human endothelial cells. Proc Nat'l Acad Sci USA 1999;96:2811-6

Moser TL, Kenan DJ, Ashley, TA, Roy JA, Goodman MD, Misra UK, Cheek DJ, Pizzo SV. Endothelial cell surface F1-F0 ATP synthase is active in ATP synthesis and is inhibited by angiostatin. Proc Nat'l Acad Sci USA 2001;98: 6656-61

Munro S. Lipid rafts: elusive or illusive? Cell 2003;115:37788

Schwiebert EM, Zsembery A. Extracellular ATP as a signaling molecule for epithelial cells. Biochim Biophys Acta 2003;1615:7-32

Sprenger RR., Speijer D, Back JW, de Koster CG, Pannekoek $\mathrm{H}$, Horrevoets AJ. Comparative proteomics of human endothelial cell caveolae and rafts using two-dimensional gel 
electrophoresis and mass spectrometry. Proteomics 2004;25: 156-72

Strohmeier GR, Lencer WI, Patapoff TW, Thompson LF, Carlson SL, Moe SJ, Carnes DK, Mrsny RJ, Madara JL. Surface expression, polarization, and functional significance of CD73 in human intestinal epithelia. J Clin Invest 1997; 99:2588-601

Wajih N, Sane DC. Angiostatin selectively inhibits signaling by hepatocyte growth factor in endothelial and smooth mus- cle cells. Blood 2003;101:1857-63

Wilson-Fritch L, Burkart A, Bell G, Mendelson K, Leszyk J, Nicoloro S, Czech M, Corvera S. Mitochondrial biogenesis and remodeling during adipogenesis and in response to the insulin sensitizer rosiglitazone. Mol Cell Biol 2003;23: 1085-94

Zhao $\mathrm{Y}$, Zhang W, Kho $\mathrm{Y}$, Zhao $\mathrm{Y}$. Proteomic analysis of integral plasma membrane proteins. Anal Chem 2004;76: 1817-23 